\title{
Preface: Algorithmic Game Theory
}

\author{
Marios Mavronicolas
}

Published online: 28 April 2011

(C) Springer Science+Business Media, LLC 2011

The nine papers in this volume were among the best papers presented at the 2 nd International Symposium on Algorithmic Game Theory (SAGT 2009) held on October 18th to 20th 2009, in Paphos, Cyprus.

The purpose of SAGT 2009 was to bring together research from Computer Science, Economics and Mathematics to present and discuss previously unpublished research results at the intersection of the Theory of Algorithms and Game Theory. Consequently, the Call for Papers to SAGT 2009 solicited new foundational work in all important areas of Algorithmic Game Theory such as:

Solution concepts in Game Theory; Game classes (e.g., bimatrix, potential, Bayesian; Exact and approximate computation of equilibria; Algorithmic Mechanism Design; Automated Mechanism Design; Convergence and learning in games; Complexity classes in Game Theory; Algorithmic aspects of fixed-point theorems; Mechanisms, incentives and coalitions; Cost-sharing algorithms and analysis; Computational aspects of market equilibria; Computational problems in Economics, Finance, Decision Theory and Pricing;

Computational social choice; Auction algorithms and analysis; Price of Anarchy and its relatives; Representations of games and their complexity; Economic aspects of Distributed Computing and the Internet; Network formation on the Internet; Congestion, routing and network design games; Game-theoretic approaches to networking problems; Byzantine Game Theory.

Those nine best papers were subsequently invited to the SAGT 2009 Special Issue of Theory of Computing Systems (TOCS). They were refereed according to the usual

M. Mavronicolas $(\bowtie)$

Department of Computer Science, University of Cyprus, 75 Kallipoleos Str., P.O. Box 20537,

1678 Nicosia, Cyprus

e-mail: mavronic@ucy.ac.cy 
standards of TOCS in a quite severe refereeing process. They were finally accepted for publication in the SAGT 2009 Special Issue.

The nine papers now published in the SAGT 2009 Special Issue focus on topics regarding (i) Scheduling Games, Congestion Games, Routing Games and Connection Games, (ii) Computational Complexity of Solution Concepts in Game Theory, (iii) the Price of Anarchy and Stability, (iv) Auctions end (v) Mechanisms.

My sincere thanks go to all authors who responded to the invitation and whose work is included in this volume. I would also like to gratefully acknowledge the immense effort of the referees who assisted my editorial work. The careful work of the referees certainly helped the authors to revise their papers so that they are now as complete as possible. 